\title{
Heuschnupfen im Mai
}

\section{Schuld kann die Eiche sein}

\author{
Ein neuer Termin sollte im Pollenflugkalender eingeschoben wer- \\ den: Zwischen der Birkenblüte im April und der Gräserblüte über- \\ wiegend von Mitte April bis Mitte Mai blüht in Deutschland die \\ Eiche. Patienten, die in dieser Zeit Heuschnupfenprobleme haben, \\ sollten daher (ergänzend) auf Eichenpollen getestet werden. Denn \\ eine Sensibilisierung gegen deren Allergene ist gar nicht so selten.
}

Pitsen ollen aus Frühblühern (Hasel, Erle, Birke), Gräsern und Getreidesorten sowie Beifuß gelten als Hauptallergene für die saisonale allergische Rhinitis. Bei vielen Allergologen gelten Sensibilisierungen außerhalb dieses Allergenspektrums als selten - obwohl Anamnese und klinische Beobachtungen bei nicht wenigen Patienten eine zeitliche Diskrepanz zwischen der Flugzeit „ihres“ Pollens und dem Auftreten der Rhinitissymptomatik erkennen lässt.

Eine Studie mit 157 Pollinosispatienten ging deshalb der Frage nach, in wie weit diese gegen Pollenarten außerhalb des heute getesteten StandardAllergenspektrums sensibilisiert waren. Bei ihnen wurde ein Hauttest mit den sechs Hauptpollenarten und zusätzlich mit Eichen-, Eschen-, Nessel-, und Wegerichextrakten durchgeführt. War der Hauttest positiv, schloss sich eine nasale Provokationstestung an.

Das Ergebnis: Rund ein Viertel (39 von 157) der Pollenrhinitiker hatte auch eine Allergie gegen Eichenpollen meist als zusätzliche Sensibilisierung. Lediglich ein Patient litt an einer isolierten Eichenpollenallergie. Bei dieser Allergie handelt es sich nicht um eine Kreuzallergie, sondern um eine eigenständige Allergisierung.

Aktuelle Sensibilisierungen gegen die drei anderen geprüften Pollenarten von Esche, Nesseln und Wegerich waren dagegen deutlich seltener.

Fazit der Autoren: Eine Eichenpollenallergie ist - zumindest im hessischen Raum - gar nicht so selten. Eine kutane Testung dieser Allergene sollte deshalb in das Standardtestprogramm aufgenommen werden. Positive Haut- reaktionen müssen allerdings, wie üblich, auf ihre klinische Relevanz hin durch nasale Provokationstests überprüft werden. Dies ist auch vor Einleitung einer Hyposensibilisierungsbehandlung zu bedenken.

\section{$b k$}

\section{Kropp R, Laumen $\mathbf{R}$}

Seltenere Pollenallergien. Allergologie

1999; 22: 611-6.

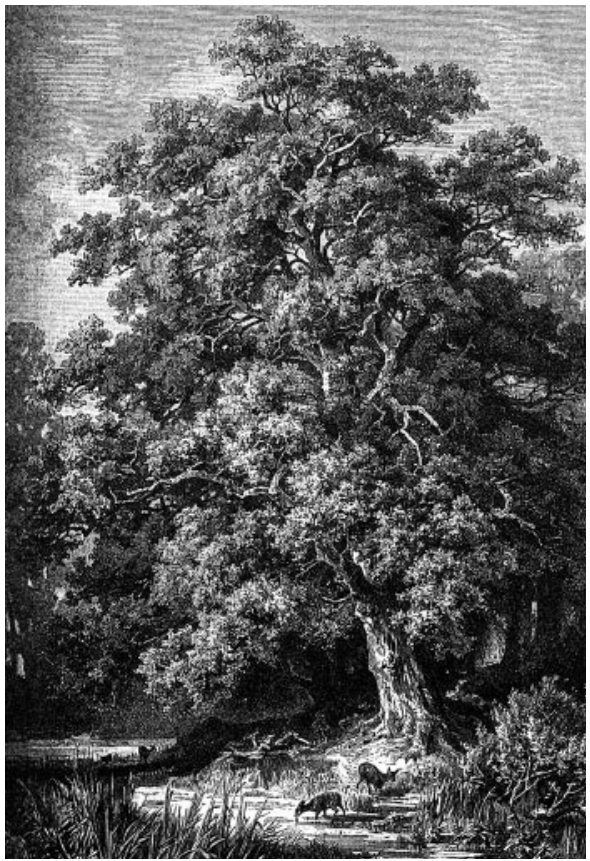

Die Pollen der Eiche können allergen wirken. Der Baum blüht ab Anfang Mai unmittelbar nach den Birken.

\section{Mit Psychotraining gegen Pollenallergie?}

Allergische Erkrankungen und psychisches Befinden stehen in enger Korrelation zueinander. Ein spezielles psychologisches Training will Pollenallergikern helfen, ihre Allergie in den Griff zu bekommen.

$\mathrm{D}^{2}$ as „Hildesheimer Gesundheitstraining", entwickelt von G. Unterberger (Hildesheim/Holzminden), vereint weiterentwickelte Methoden der Hypnose und Verhaltenstherapie sowie des neurolinguistischen Programmierens. So sollen sich Birkenpollenallergiker z.B. vorstellen, sie gingen durch eine Pappelallee ohne Birken und ihr Immunsystem reagiere ganz normal - eben gar nicht. Das Immunsystem lerne dabei, so die Hypothese, seine Überreaktion auf Birkenpollen abzulegen. Weiterhin soll auch die Vorstellung hilfreich sein, man befinde sich hinter einer gläsernen Wand, die vor Pollen schützt.

73 Patienten mit einer Allergie gegen Birkenpollen wandten diese Methode über 2 Monate an. Der Erfolg ist erstaunlich: Während zu Beginn des Gesundheitstrainings bereits z.B. die die Vorstellung eines Spaziergangs durch einen Birkenhain zumindest bei einigen Patienten ausreichte, eine allergische Reaktion auszulösen, hatten die meisten Probanden am Ende des Trainings gelernt, mentale Schutzmaßnahmen gegen die allergische Reaktion zu aktivieren. Das Immunsystem, das irrtümlich gelernt habe, Birkenpollen immunologisch abzuwehren, werde durch das veränderte innere Erleben „umprogrammiert" - so die Forscher. Bei $80 \%$ der Studienteilnehmer nahmen die allergischen Reaktionen ab oder verschwanden sogar ganz, der Medikamentenverbrauch sank.

$\mathrm{Ob}$ andere Arbeitsgruppen eine ähnlich hohe Erfolgsquote mit dieser Methode erzielen, sei dahin gestellt. $b k$

\section{Pressemitteilung des pid Presseinfo-} Dienstes, 1999. 